\title{
Numerical Simulating Nonlinear Effects of Ultrasonic Propagation on High-speed Ultrasonic Gas Flow Measurement
}

\author{
Li Yue-zhong ${ }^{1,2, *}$, Wu Jiang-tao ${ }^{1}$ and Hu Kai-ming ${ }^{2}$ \\ ${ }^{1}$ School of Energy and Power Engineering, Xi 'an Jiaotong University, Xi ' an 710049, China \\ ${ }^{2}$ School of Mechanical and Electronic Engineering, East China Institute of Technology, Nanchang 330013, China
}

Received: 28 Jan. 2013, Revised: 29 May. 2013, Accepted: 30 May. 2013

Published online: 1 Sep. 2013

\begin{abstract}
The effects of nonlinear ultrasonic propagation on high-speed ultrasonic gas flow measurement are analyzed based on the sound line equation derived from Snell's geometric acoustic law. A mathematical model for the ultrasonic propagation path in ultrasonic flowmeter pipe is built, and the relationship between $\mathrm{x}$ and $\mathrm{r}$ is calculated by MATLAB programming and ode 45 simulating. The ultrasonic propagation path at the flow rate of $3 \sim 30 \mathrm{~m} / \mathrm{s}$ is simulated in the assumed boundary conditions of the pipeline, transducer installation and fluid state. It shows that the nonlinear propagation characteristics cause the large deviations of the position of received ultrasonic waves in conditions of different flow rates, which strongly affects the stability and accuracy of flow measurement. The simulated offset data of the receiving position are useful for the high-speed gas ultrasonic flowmeter installation and dry calibration on clamp-on ultrasonic flowmeters.
\end{abstract}

Keywords: Numerical Simulating, Ultrasonic Propagation, Ultrasonic Gas

\section{Introduction}

As a non-intrusive flow measurement device, the ultrasonic flowmeter has a unique advantage in flammable and explosive gas flow measurement. In the large-diameter pipe natural gas transmission industry, the pipeline flow rate in medium-pressure gas transmission is mostly restricted to $10 \mathrm{~m} / \mathrm{s}$ or so. In order to improve gas transport efficiency, the natural gas transmission speed is increased to $15 \mathrm{~m} / \mathrm{s}$ $\sim 20 \mathrm{~m} / \mathrm{s}$ under $0.2 \mathrm{MPa} \sim 0.4 \mathrm{MPa}$, the steam transmission speed is mostly controlled in the range of $30 \mathrm{~m} / \mathrm{s} \sim$ $40 \mathrm{~m} / \mathrm{s}$, and the superheated steam flow rate is more than $60 \mathrm{~m} / \mathrm{s}$. Hydrogenation in the hydrogen stations generally uses small diameter pipe $(15 \mathrm{~mm} \sim 18 \mathrm{~mm})$ at the pressure of $30 \mathrm{MPa}$ or more, and the hydrogen flow rate is more than $65 \mathrm{~m} / \mathrm{s}$. Recently, the hydrogenation pressure is increased to $70 \mathrm{MPa}$ or $75 \mathrm{MPa}$, and the calculated hydrogen flow rate is $120 \mathrm{~m} / \mathrm{s}$ or more. However, due to the effects of the flow rate on nonlinear ultrasonic propagation, most of the ultrasonic gas flowmeters' measurement range is less than $30 \mathrm{~m} / \mathrm{s}$. This paper mainly considers the application of the ultrasonic flowmeter for high-speed gas flow mea- surement. At first, the ultrasonic propagation characteristics in the high-speed gas medium are analyzed, then, the effects of the flow rate on nonlinear ultrasonic propagation are simulated to provide the theoretical and methodological bases to develop the high-speed gas ultrasonic flowmeter.

Some researchers have studied the nonlinear characteristics of ultrasonic propagation. Vanhille et al. [1] simulated the nonlinear ultrasound propagation properties in the fluid with the Lagrangian equation, and they mainly studied the nonlinear relationship between the sound pressure and the propagation distance. Willatzen et al. [2,3] studied the non-linear relationship between the propagation time and the flow rate based on the fluid dynamic equations and the basic formula for measuring the flow rate. Q. Ji et al. [4-9] studied the ultrasonic propagation characteristics and model in aluminum, colloids and other materials. Francesco Lanza di Scalea et al. [10] observed and measured the ultrasonic propagation characteristics

through establishing the physical experimental apparatus and mainly studied the relationship between the re-

\footnotetext{
*Corresponding author e-mail: li-yuezhong@ sohu.com
} 
ceived echo signal and the propagation characteristics. The American Gas Association (AGA) and the China's Administration of Quality Supervision, Inspection and Quarantine (AQSIQ) [11,12] have established successively the relevant standards of gas and natural gas flow measurement for ultrasonic flowmeters, in which the ultrasound propagates in the pipeline from the emitting transducer to the receiving by the S-shaped path, and the ultrasonic propagation equation determined by Snell's law is presented roughly. However, a few papers have been reported about the relationship between the nonlinear ultrasonic propagation path and the flow rate. So, this work mainly deals with the effects of flow rates on ultrasonic propagation path based on the ultrasonic propagation equation determined by Snell's law and the wave propagation trajectory equation derived by Boone and Vermaas.

\section{Ultrasonic propagation model in the ultrasonic flowmeter pipe}

The structure of the typical clamp-on ultrasonic flowmeter investigated in this work is shown in Fig.1 [13], and it consists of clamp holder, upstream and downstream transducers, preamplifier, and ultrasonic flowmeter. It is the typical structure of the small diameter clamp-on ultrasonic flowmeters in American GE, German Flexim, Japan's Fuji Corp etc. According to GB/TI8604-2001 and AGA Report No.9, the average axial flow velocity formula for transittime single path ultrasonic flowmeter is:

$$
V=K_{C} \bullet \frac{L}{2 \cos \varphi_{0}} \bullet \frac{t_{U}-t_{D}}{t_{D} t_{U}}
$$

The related parameters are shown in Fig.2, where, $L$ is the straight line length between the ultrasonic sensors $A$ and $B$, and $\phi_{0}$ is the initial transmission angle for the ultrasonic path. In equation $(1), t_{D}$ is the ultrasonic downstream propagation time, $t_{U}$ is the ultrasonic upstream propagation time, and $K_{c}$ is the velocity distribution coefficient.

As the flow rate along the pipe cross-section is not constant, the ultrasonic wave propagation path in the pipeline is not linear. According to velocity distribution function $V(r)$ and Snell's geometrical acoustic law, the ultrasonic transmission line equation is determined as

$$
\frac{C}{\cos \varphi(r)}+V(r)=\text { Const }
$$

Where, $C$ is the ultrasonic propagation velocity in air.

According to Boone and Vergas's model, the acoustic line equation is written as:

$$
\left\{\begin{array}{l}
\frac{d x}{d t}=C \times \cos \varphi(r)+V(r) \\
\frac{d r}{d t}=C \times \sin \varphi(r) \\
\frac{d \varphi(r)}{d t}=-\cos ^{2} \varphi(r) \frac{d V(r)}{d r}
\end{array}\right.
$$

Where, $x$ is the ultrasonic propagation horizontal displacement from the transmitting sensor as a starting point, $r$ is the ultrasonic propagation radial displacement, and $\phi(r)$ is the angle between the particle motion tangent and the axis in position $r$ of ultrasonic propagation path.

According to the empirical formula of Prandtl velocity distribution, and considering the fully developed turbulent flow, the velocity distribution is:

$$
V(r)=V_{m}\left(1-\frac{|r|}{R}\right)^{\frac{1}{n}}
$$

Where, $V_{m}$ is the surface velocity along the axial line, and $R$ is the radius of the pipeline. Considering only the smooth pipe, the index $n$ is:

$$
n=2 \log _{10}\left(\frac{R_{e}}{n}\right)-0.8,
$$

Where, $R e$ is Reynolds number of fluid in the pipelinewhich is defined as $R_{e}=\frac{V D}{v}$, and $v$ is the motion viscosity.

The mean flow velocity $V_{z}$ on the vertical center parallel pipeline section and the pipeline mean flow velocity $V$ both are defined respectively as

$$
\begin{aligned}
& V_{Z}=\frac{1}{L} \int_{L} V(r) d L, \\
& V=\frac{1}{S} \iint_{S} V(r) d S,
\end{aligned}
$$

Where, $L$ is the ultrasonic path length; $S$ is the pipeline inner sectional area. So the relation of $V_{z}$ and $V$ is:

$$
V=\frac{2 n}{(2 n+1)} V_{Z},
$$

Eq.(8) is compared with Eq.(1), and there are definitions as follows

$$
\begin{gathered}
K_{C}=\frac{2 n}{(2 n+1)}, \\
V_{Z}=\frac{L}{2 \cos \varphi_{0}} \bullet \frac{t_{U}-t_{D}}{t_{D} t_{U}},
\end{gathered}
$$

From Eq.(3) and Eq.(4), according to the downstream and upstream situations a model is established as follows:

(1) Ultrasonic propagation in the downstream direction The relationship of $\phi$ and $r$ can be derived as:

$$
\left\{\begin{array}{l}
\frac{d(r)}{d(\varphi)}=\frac{-n R c \sin \varphi}{v_{m} \cos ^{2} \varphi\left(1-\frac{r}{R}\right)^{\frac{1}{n}-1}} 0 \leqslant r \leqslant R \\
\frac{d(r)}{d(\varphi)}=\frac{-n R c \sin \varphi}{v_{m} \cos ^{2} \varphi\left(1+\frac{r}{R}\right)^{\frac{1}{n}-1}}-R \leqslant r \leqslant 0
\end{array},\right.
$$

And the relationship of $x$ and $r$ is obtained as:

$$
\left\{\begin{array}{l}
d(r)=\frac{-c \sin \varphi}{v_{m}\left(1-\frac{r}{R}\right)^{\frac{1}{n}}+c \cos \varphi} d(x) \quad 0 \leqslant r \leqslant R \\
d(r)=\frac{c \sin \varphi}{v_{m}\left(1+\frac{r}{R}\right)^{\frac{1}{n}}+c \cos \varphi} d(x)-R \leqslant r \leqslant 0
\end{array},\right.
$$


(2) Ultrasonic propagation in the upstream direction The relationship of $\phi$ and $r$ can be derived as:

$$
\left\{\begin{array}{l}
\frac{d(r)}{d(\varphi)}=\frac{n R c \sin \varphi}{v_{m} \cos ^{2} \varphi\left(1+\frac{r}{R}\right)^{\frac{1}{n}-1}}-R \leqslant r \leqslant 0 \\
\frac{d(r)}{d(\varphi)}=\frac{n R c \sin \varphi}{v_{m} \cos ^{2} \varphi\left(1-\frac{r}{R}\right)^{\frac{1}{n}-1}} 0 \leqslant r \leqslant R
\end{array},\right.
$$

And the relationship of $x$ and $r$ is obtained as

$$
\left\{\begin{array}{l}
d(r)=\frac{-c \sin \varphi}{v_{m}\left(1+\frac{r}{R}\right)^{\frac{1}{n}}+c \cos \varphi} d(x)-R \leqslant r \leqslant 0 \\
d(r)=\frac{c \sin \varphi}{v_{m}\left(1-\frac{r}{R}\right)^{\frac{1}{n}}+c \cos \varphi} d(x) \quad 0 \leqslant r \leqslant R
\end{array}\right.
$$

\section{Simulation and analysis of models}

\subsection{Method}

According to Eq. (11) and Eq. (13), the results can be derived as follows:

(1) Ultrasonic propagation in the downstream direction:

$$
\left\{\begin{array}{l}
\cos \varphi=\frac{c}{c \sec \varphi_{0}-R v_{m}\left(1-\frac{r}{R}\right)^{\frac{1}{n}}} 0 \leqslant r \leqslant R \\
\cos \varphi=\frac{c}{c \sec \varphi_{0}-R v_{m}\left(1+\frac{r}{R}\right)^{\frac{1}{n}}}-R \leqslant r \leqslant 0
\end{array},\right.
$$

(2) Ultrasonic propagation in the upstream direction:

$$
\left\{\begin{array}{l}
\cos \varphi=\frac{c}{c \sec \varphi_{0}+R v_{m}\left(1+\frac{r}{R}\right)^{\frac{1}{n}}}-R \leqslant r \leqslant 0 \\
\cos \varphi=\frac{c}{c \sec \varphi_{0}+R v_{m}\left(1-\frac{r}{R}\right)^{\frac{1}{n}}} 0 \leqslant r \leqslant R
\end{array},\right.
$$

When Eq. (15) is put into (12), and (16) into (14), a complex equation can be derived, and it is difficult to solve the integral directly. With the numerical solution method of ordinary differential equation MATLAB ode45, the relationship of $x$ and $r$ can be solved. Using the Runge-Kutta algorithm, ode45 is a variable step size solver. Ode45 has fourth-order and fifth-order Runge-Kutta single-step algorithm. Its truncation error is $(\Delta x) 3$. It can be mainly used to solve Nonstiff (non-rigid) of ordinary differential equations, and it is the preferred method to solve the problem of numerical solution.

\subsection{Discussion}

The measurement pipeline condition is assumed as $R=$ $100 \mathrm{~mm}$ and $c=340 \mathrm{~m} / \mathrm{s}$, and the initial ultrasound launch angle is $\phi_{0}=30^{\circ}$.

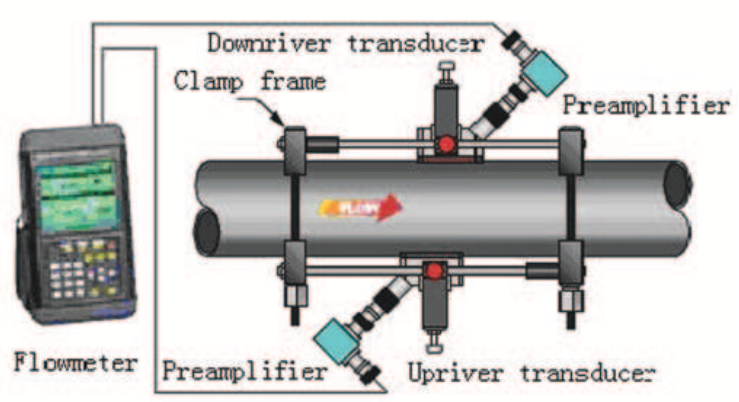

Figure 1 clamp-on ultrasonic flowmeter.

If $R e=4 e 6, n=10.3724$.

The ultrasonic propagation trajectory under

$V=\{12,15,18,24,27,30\}(\mathrm{m} / \mathrm{s})$

is calculated and depicted by calculating and simulating with MATLAB and ode45.

Fig.3 and Fig.4 show respectively ultrasound downstream and upstream transmission paths at higher flow rates. In order to show the level migration of ultrasonic wave reaching the end of the pipeline wall at the different flow rates more clearly, Fig. 3 and 4 are respectively enlarged partly as shown in Fig. 5 and 6.

In Fig. $3 \sim$ Fig.6, the solid line is the ultrasonic propagation path at zero fluid velocity. The others are the ultrasonic propagation paths from the left to the right at $V=$ $\{12,15,18,24,27,30\}(\mathrm{m} / \mathrm{s})$. As can be seen from Fig. 3 and Fig. 5, the deviation from the zero velocity of ultrasonic downstream propagation paths is larger with the higher flow rate. As shown in Fig. 4 and Fig. 6, the deviation from the zero velocity of ultrasonic upstream propagation paths is larger too with the higher flow rate. However, as presented in Fig. 5 and Fig. 6, the downstream offset is larger clearly than the upstream one, and is about 2 times that of the upstream.

In order to compare the offsets of ultrasonic propagation path to reach the end of the pipeline wall under the lower flow rates, Fig. 7 and Fig. 8, show propagation paths of the ultrasonic wave respectively calculated by MATLAB and ode 45 at $V=3 \sim 12 \mathrm{~m} / \mathrm{s}$ in downstream and upstream. Fig. $3 \sim 8$ shows that the maximum offsets of the pipeline end of ultrasonic propagation path under the high speed fluid are 2 times or more than under the zero speed fluid. Through calculating by MATLAB programming, when $V=30 \mathrm{~m} / \mathrm{s}$, the displacement of ultrasonic downstream propagation in $x$ direction is obtained, i.e. $X_{30}=$ $0.363 \mathrm{~m}$. When $V=0 \mathrm{~m} / \mathrm{s}$, the displacement is obtained, i.e. $X_{0}=0.346 m$ and then the offset of ultrasonic downstream propagation is $\Delta x=X_{30}-X_{0}=0.015 \mathrm{~m}$. The same method can be used to calculate the offset of ultrasonic upstream propagation, that is, $\Delta x_{U}=0.007 \mathrm{~m}$. By this way, the above conclusion is verified. 


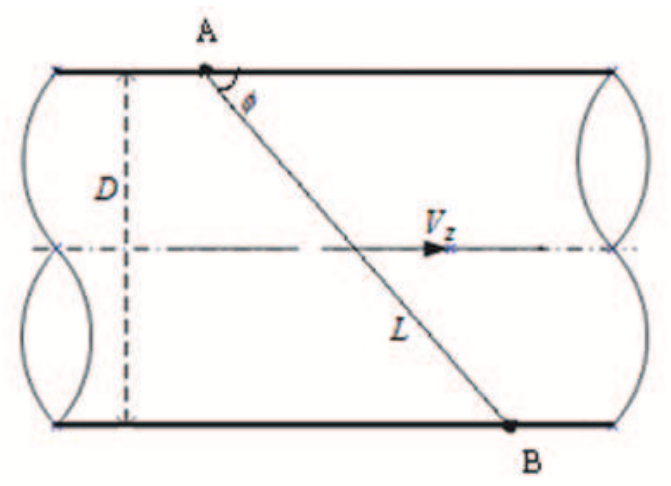

Figure 2 ultrasonic propagation in the pipeline.

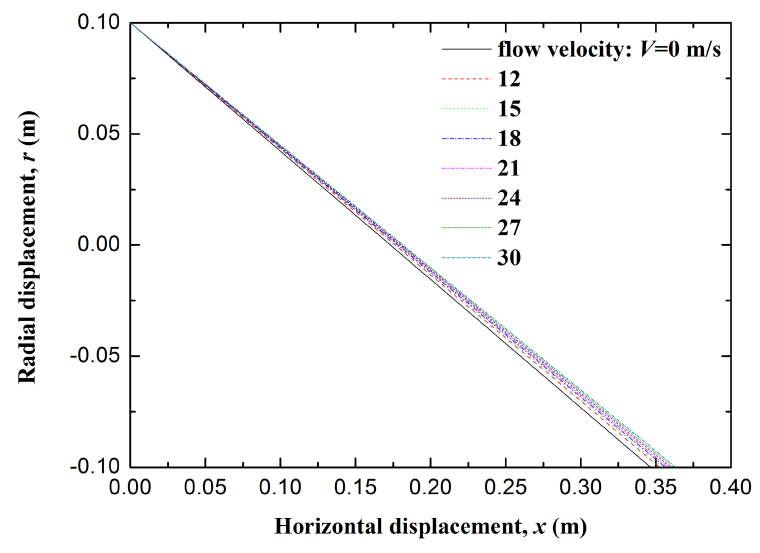

Figure 3 ultrasonic downstream propagation path in Highvelocity fluids.

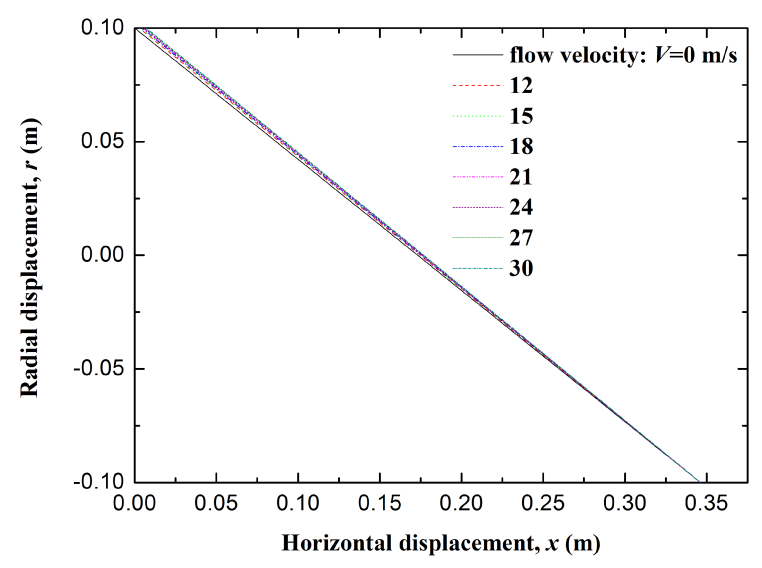

Figure 4 ultrasonic upstream propagation path in High-velocity fluids.

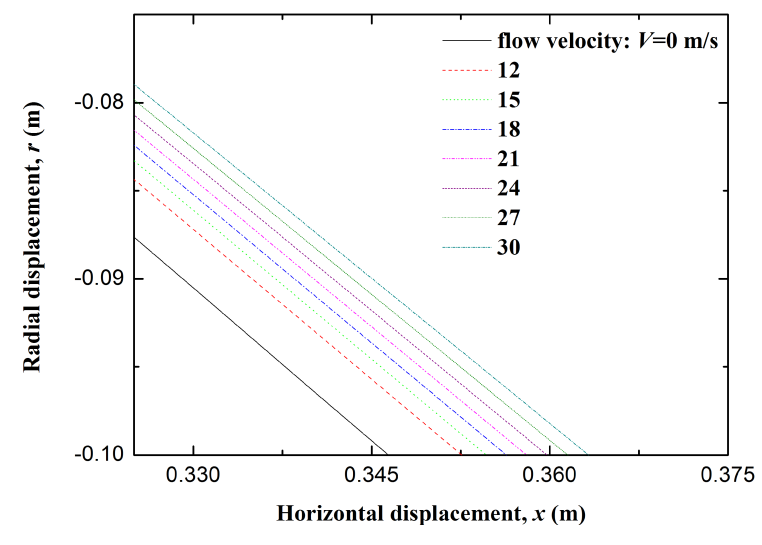

Figure 5 the enlargement diagram of ultrasonic downstream propagation path in High-velocity fluids.

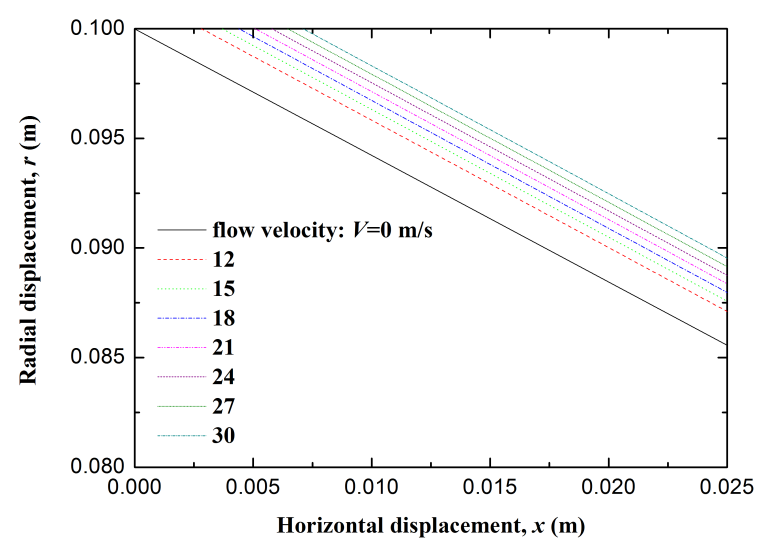

Figure 6 the enlargement diagram of ultrasonic upstream propagation path in High-velocity fluids.

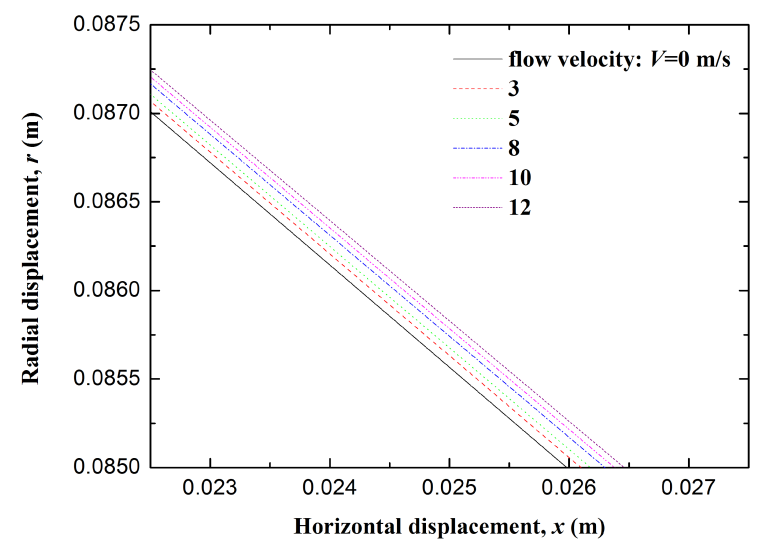

Figure 7 the enlargement diagram of ultrasonic downstream propagation path in Low-velocity fluids. 


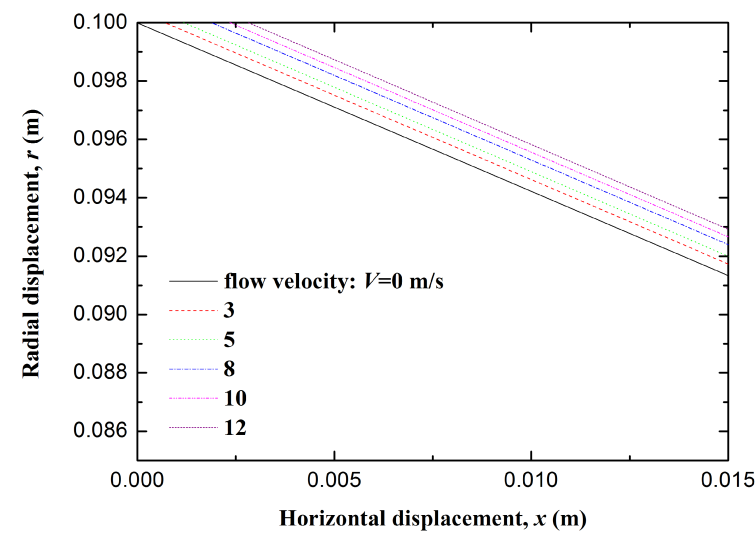

Figure 8 the enlargement diagram of ultrasonic upstream propagation path in Low-velocity fluids.

\section{Conclusion}

Theoretically, with analysis on nonlinear character of the ultrasonic propagation in gas flow measurement, we have drawn the conclusion that the nonlinear ultrasonic propagation causes a deviation of ultrasonic wave reaching the end of the pipeline wall from the receiving ultrasonic sensor. Since the position of received ultrasonic wave changes at the flow rate, the position will deviate from the center of the receiving ultrasonic sensors' effective area (usually with the diameter of $7 \sim 12 \mathrm{~mm}$ ), especially at the higher flow rate. The propagation time also changes with the flow rate, which affects the accuracy of the ultrasonic flowmeter. According to the above simulation results, the maximum offset is $15 \mathrm{~mm}$ at the flow rate $\mathrm{V}=30 \mathrm{~m} / \mathrm{s}$, and will be even greater for the higher speed fluid. So, the large-diameter insert-type ultrasonic gas flowmeter can be usually used at the occasions of the relatively steady flow field and flow velocity. And for the clamp-on ultrasonic gas flowmeter, two movable ultrasonic sensors can be designed to improve the flow measurement stability and accuracy. The above simulating results are very useful for the high-speed ultrasonic gas flowmeter installation and the dry calibration on clamp-on ultrasonic flowmeters.

\section{References}

[1] C. Vanhille, C. Campos-Pozuelo. A numerical formulation for nonlinear ultrasonic waves propagation in fluids. Ultrasonics 2004, 42, 1123-1128 (2004)

[2] M. Willatzen, H. Kamath. Nonlinearities in ultrasonic flow measurement. Flow Measurement and Instrumentation, 19, 223-232 (2008).

[3] H. M. Habib, E. R. El-Zahar. A New Algorithm for Solving Nonlinear Stationary Shock Problems with Mechanization Applied Mathematics \& Information Sciences, 1, 31-45 (2010).
[4] Q. Ji, L. H. Le, L. J. Filipow, etc. Ultrasonic wave propagation in water-saturated aluminum foams. Ultrasonics, 36, 759-765 (1998).

[5] J. S. Tebbutt, R. E. Challis. Ultrasonic wave propagation in colloidal suspensions and emulsions: a comparison of four models. Ultrasonics, 34, 363-368 (1996).

[6] B. C. Lee, M. Palacz, M. Krawczuk, etc.Wave propagation in a sensor/actuator diffusion bond model. JOURNAL OF SOUND AND VIBRATION, 276, 671-687 (2004).

[7] P. P. Delsanto, Sigrun Hirsekorn, V. Agostini, etc. Modeling the propagation of ultrasonic waves in the interface region between two bonded elements. Ultrasonics, 40, 605-610 (2002).

[8] Zhang Rongxin, Qin Guoliang, Xu Lina. High-accuracy numerical simulation of acoustic propagation problem in pipe, Journal of Vibration and Shock, 29, 115-119 (2010).

[9] Francesco Lanza di Scalea, Robert E. Green Jr. Experimental observation of the intrusive effect of a contact transducer on ultrasound propagation. Ultrasonics, 37, 179-183 (1997).

[10] Yuto Inoue, Hiroshige Kikura, Hideki Murakawa etc. A study of ultrasonic propagation for ultrasonic flow rate measurement. Flow Measurement and Instrumentation, 19, 223232 (2008).

[11] American Gas Association.A.G.A.Report No.9: Measurement of Gas by Multipath ultrasonic Meters, (1998).

[12] Chinese General Administration of Quality Supervision, Inspection and Quarantine. Ultrasonic gas flowmeter measuring gas flow (GB/TI8604-2001). Beijing: Standards Press of China, (2001).

[13] GE Sensing \& Inspection Technologies. Ultrasonic flowmeter. http://www.gesensing.com.

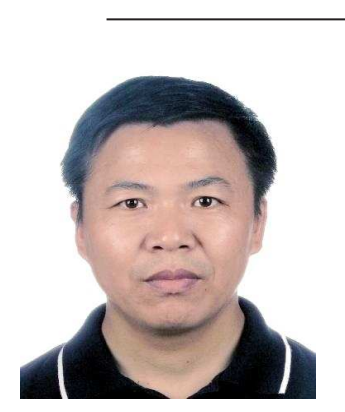

\section{Li Yuezhong}

received the $\mathrm{PhD}$. in Detection technology and automation equipment from Huazhong University of Science and Technology in 2010, and he is as a postdoctoral research fellow in $\mathrm{Xi}$ 'an Jiaotong University. He is currently an associate professor in East China Institute of Technology. His research interest is in the areas of ultrasonic flow measurement, and intelligent instrumentation.

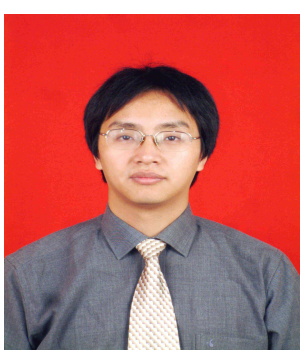

\section{Wu Jiangtao} is a professor and doctoral tutor in $\mathrm{Xi}$ ' an Jiaotong University. He is a Fellow of IUPAC (International Union of Pure and Applied Chemistry)and associate Editor of Journal of Chemical and Engineering Dataand a member of Advisory Board of the Journal of Chemical Thermodynamics. He does research on thermophysical properties Molecular simulation of fluidsdatabase of thermophysical properties based on the Web and alternative refrigerants and clean fuels. 